\title{
Low Mass X-ray Binaries and Globular Clusters in Early-Type Galaxies
}

\author{
Craig L. Sarazin \\ Department of Astronomy, University of Virginia, Charlottesville, VA 22903-0818, USA \\ email: sarazin@virginia.edu
}

\begin{abstract}
Chandra observations have allowed the detection of a large number of low mass Xray binaries (LMXBs) in early-type galaxies. Comparison to catalogs of globular clusters (GCs) from Hubble Space Telescope observations have shown that a high fraction of the LMXBs in early-type galaxies are associated with GCs. The fraction of LMXBs associated with globular clusters increases along the Hubble sequence from spiral bulges to S0s to Es to cDs. On the other hand, the fraction of globular clusters which contain X-ray sources appears to be roughly constant ( $\sim 4 \%$ for $L_{X} \gtrsim 10^{38} \mathrm{ergs} / \mathrm{s}, \sim 10 \%$ for $L_{X} \gtrsim 10^{37} \mathrm{ergs} / \mathrm{s}$ ). There is a strong tendency for the X-ray sources to be associated with the optically more luminous GCs. There is a trend for the $\mathrm{X}$-ray sources to be found preferentially in redder, more metal-rich GCs, which is independent of optical luminosity correlation.

The relative role of formation of LMXBs in GCs and in situ formation in the field is uncertain. One of the best ways to study this is to compare the spatial distribution of GC-LMXBs, field LMXBs, GCs, and optical light in the galaxies. Theoretical models and results of fits to the observed distributions are presented.
\end{abstract}

Keywords. binaries: close, galaxies: elliptical and lenticular, galaxies: star clusters, galaxies: individual (NGC 4365), globular clusters: general, X-rays: binaries, X-rays: galaxies.

\section{Introduction}

Chandra observations have resolved most of the X-ray emission in X-ray-faint earlytype galaxies into individual point-like sources (e.g., Sarazin, Irwin, \& Bregman 2000). Given their properties and the stellar populations in these galaxies, these X-ray sources are assumed to be Low Mass X-ray Binaries (LMXBs). A significant fraction ( $20-70 \%)$ of the LMXBs are associated with globular clusters in the host galaxies (Sarazin et al. 2001; Angelini et al. 2001). The fraction of LMXBs located in GCs is much higher than the fraction of optical light, which indicates that stars in GCs are much more likely (by a factor of $~ 500$ ) to be donor stars in X-ray binaries than field stars. As has been known for a number of years, a similar result applies to our own Galaxy and to the bulge of M31 (e.g., Hertz \& Grindlay 1983). This is generally believed to result from stellar dynamical interactions in globular clusters, which can produce compact binary systems.

X-ray observations with $A S C A$ indicated that the total luminosity of LMXBs in earlytype galaxies correlated better with the number of GCs than with the optical luminosity of the galaxy (White et al. 2002). This is somewhat surprising, as a nontrivial fraction $(\sim 50 \%)$ of the LMXBs in most of the early-type galaxies observed so far with Chandra are not identified with GCs. This suggests that most (perhaps all?) of the LMXBs in early-type galaxies were made in GCs (Grindlay 1984; Sarazin et al. 2001; White et al. 2002). The field LMXBs might have been ejected from globular clusters individually by stellar dynamical processes (or possibly by supernova kicks), or emerged when globular clusters were destroyed by tidal effects. Alternatively, the field LMXBs may have been made in situ from primordial binaries. 

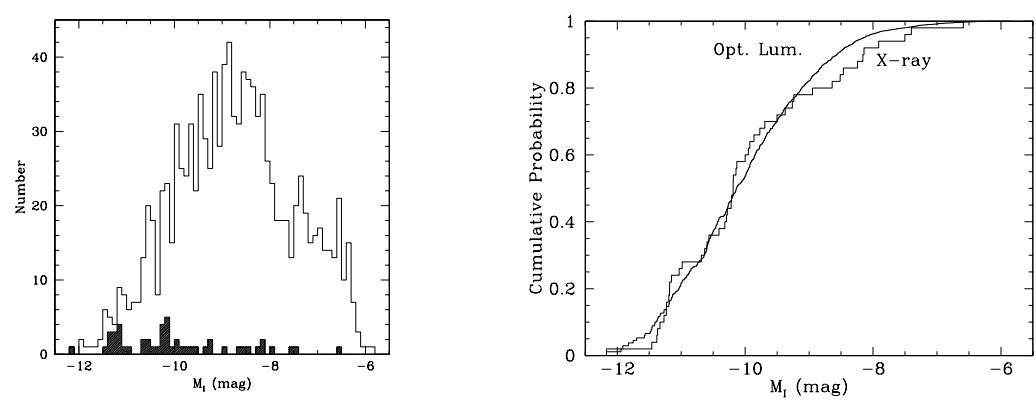

Figure 1. (a) Histograms of the number of globular clusters versus their absolute magnitude $M_{I}$ in a sample of galaxies with Chandra data (Sarazin et al. 2003). The upper histogram is for all of the GCs in the galaxies. The lower shaded histogram shows the GCs which contain identified LMXBs. (b) Cumulative distribution functions for the probability that a GC contains an X-ray source ("X-ray") and for the optical luminosity of GCs ("Opt. Lum.").

\section{Statistics of LMXBs and GC Populations}

The fraction of LMXBs in a galaxy which are associated with GCs increases along a Hubble sequence from spiral bulges $(\sim 10 \%)$ to S0s $(\sim 20 \%)$ to giant ellipticals $(\sim 50 \%)$ to $\mathrm{cD}$ galaxies $(\sim 70 \%)$ (Sarazin et al. 2003$)$. There is a well-established trend for the specific frequency of GCs in galaxies ( $S_{N}$, the number of GCs per optical luminosity) to increase along the same Hubble sequence (e.g., Harris 1991). The detailed correlation of the fraction of LMXBs in GCs with $S_{N}$ is more consistent with most of the field LMXBs being made in situ in the field (Juett 2005; see also Sarazin et al. 2003; Maccarone et al. 2003) On the other hand, Irwin (2005) argued recently that a significant portion of the field LMXBs in S0 galaxies may have come from disrupted GCs.

The fraction of globular clusters which contain X-ray sources appears to be roughly constant from galaxy to galaxy. For samples of LMXBs with a high limiting X-ray luminosity, $L_{X} \gtrsim 10^{38} \mathrm{ergs} / \mathrm{s}$, the fraction is $\sim 4 \%$ (Kundu et al. 2002; Sarazin et al. 2003). For NGC 4697, a nearby elliptical with deep X-ray and GC observations, the fraction reaches $\sim 10 \%$ for $L_{X} \gtrsim 10^{37} \mathrm{ergs} / \mathrm{s}$ (Sivakoff et al. 2005).

\section{Properties of GCs Containing LMXBs}

Figure 1(a) shows histograms of the absolute I magnitude, $M_{I}$, of the total GC sample (upper histogram) and of the GCs containing LMXBs (shaded histogram). The LMXBs seem to be associated preferentially with the more optically luminous GCs (Angelini et al. 2001; Kundu et al. 2002; Sarazin et al. 2003). For example, the median value of $M_{I}$ for non-X-ray GCs is -8.7 , while the corresponding value for the X-ray GCs is -10.2. Using the Wilcoxon or equivalent Mann-Whitney rank-sum tests, the distribution of X-ray and non-X-ray GC luminosities are found to disagree at more than the $6 \sigma$ level.

Of course, a correlation between optical luminosity and the probability of having an X-ray source is not unexpected. LMXBs contain normal stars, and globular clusters which have higher luminosities have more stars as potential donors in LMXBs. Thus, it is interesting to test the hypothesis that the probability that a GC contains a LMXB is proportional to its optical luminosity. Figure $1(b)$ compares the cumulative probability distribution of LMXBs versus the cumulative distribution of the optical luminosity in GCs. The two cumulative distribution functions track one another fairly well. For example, half of the optical luminosity comes from GCs brighter than $M_{I}=-10.1$, while the medium absolute magnitude of GCs with LMXBs is -10.2 . The KS two-sample test was used to compare the two distributions; they are not significantly different. Thus, the 
current data indicate that optically bright GCs are much more likely to contain LMXBs than faint GCs, but the distribution is consistent with a constant probability per unit optical luminosity (Kundu et al. 2002; Sarazin et al. 2003). Recently, Jordán et al. (2004) found a correlation between the density of stars in M87 GCs and the occurrence of LMXBs. This would be consistent with the formation of LMXBs by dynamical collision processes in GCs, although the detailed form of the correlation found by Jordán et al. (2004) was also nearly equivalent to a simple dependence on the number of stars.

Figure 2 shows histograms of the $V-I$ colors for the total GC sample (upper histogram) and for the GCs containing LMXBs (shaded histogram). Because the sample contains a number of different galaxies, the overall color distribution may be less obviously bimodal than that seen in some individual galaxies. The LMXBs appear to be associated preferentially with the redder GCs (larger values of $V-I$ ) (Angelini et al. 2001; Kundu et al. 2002; Sarazin et al. 2003). The median color of the non-X-ray GCs is $V-I=1.07$, while the corresponding median for the X-ray GCs is 1.14. Using the Wilcoxon or MannWhitney rank-sum tests or the KS test, the probabilities that the two color distributions where drawn from the same distribution are $<0.2 \%$. Roughly, red GCs are three times as likely to harbor a LMXB as blue GCs.

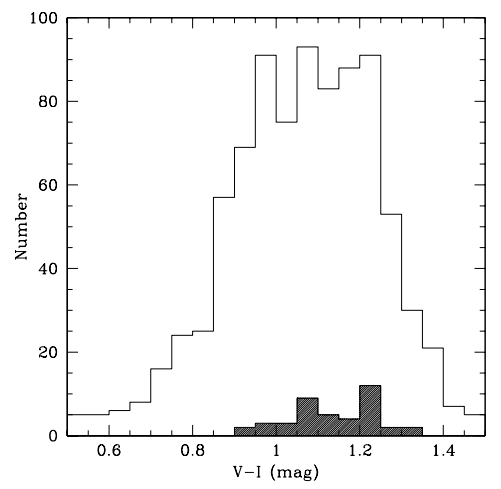

Figure 2. Histograms of the number of GCs versus their optical color, $V-I$. The upper histogram is for all of the GCs, while the lower shaded histogram shows the GCs which contain LMXBs.

\section{Spatial Distributions of Stars, GCs, and LMXBs}

The radial distribution of GCs in elliptical galaxies is more extended than that of the field stars; in particular, the optical light profiles of ellipticals typically show a central cusp, whereas the spatial distribution of GCs has a constant surface density core. This may indicate that GCs never formed in the central regions, or that the GCs which were initially formed there were disrupted by tidal effects. One way to test this would be to search the central regions of ellipticals for a stellar population which is characteristic of GCs. This is difficult for optical stars; however, as noted above, LMXBs are preferentially produced in GCs. At the same time, we would like to know what fraction of the field LMXBs were made in GCs. Some of the GC LMXBs might have escaped individually due to stellar dynamical interactions, or they may have been released when their host GC was disrupted by tidal effects. In either case, these field LMXBs would have a spatial distribution which reflected the initial spatial distribution of the GCs. Thus, by studying the spatial distributions of the optical light, GCs, and field and GC LMXBs in galaxies, we can constrain models for the formation and destruction of GCs and the origin of LMXBs.

Figure 3 shows models for the spatial distribution of field and GC LMXBs in an elliptical galaxy (Sarazin 2005). The stellar and GC distribution were based on observations of NGC 4365, and the X-ray sources are from our earlier Chandra observation of this galaxy. Here, I show only three extreme models. In Model 1, all field LMXBs were made in situ. This model probably provides an adequate fit to the existing data. Note the general result that the observed LMXB distribution is broader than that of the stars, reflecting the contribution of GC LMXBs. In Model 2, all LMXBs are made in GCs, and the field LMXBs were individually ejected from GCs. In this model, the predicted 

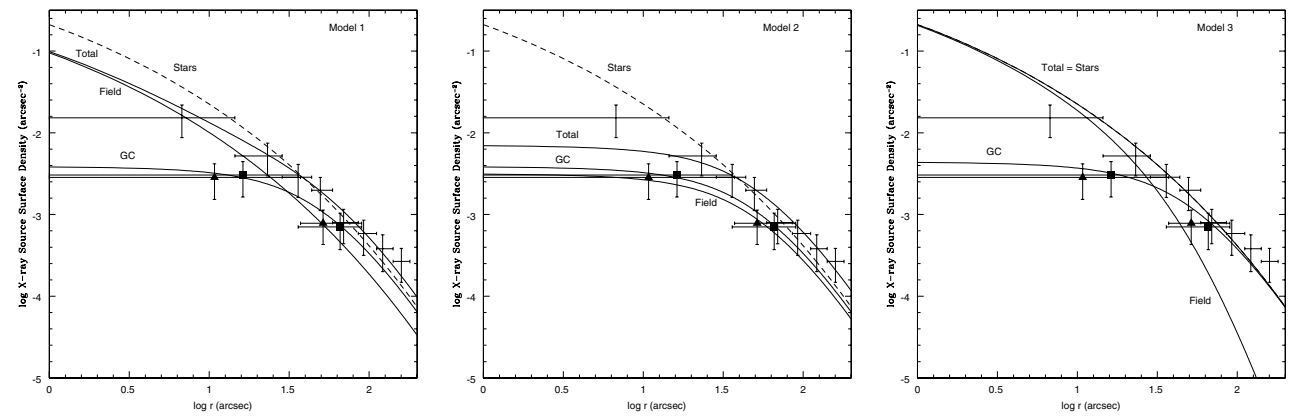

Figure 3. Predicted surface density distributions of X-ray sources in elliptical galaxies (Sarazin 2005). The solid curves labeled GC, Field, and Total show the surface densities of LMXBs in GCs, in the field, and in total, respectively. The dashed curve shows the total X-ray distribution if the X-ray sources followed the distribution of field stars. The data points are from NGC 4365. The error bars with triangles are the field LMXBs, those with squares are the GC sources, and the plain error bars are the total source distribution. Model 1 assumes that no GC LMXBs are lost from GCs either due to individual ejection or GC destruction. In Model 2, all LMXBs are made in GCs, no GCs are destroyed, but all of the field LMXBs were individually ejected from GCs. In Model 3, all LMXBs are made in GCs, and all field LMXBs result from the tidal disruption of GCs.

distribution of the LMXBs is broader than that observed. Finally, in Model 3, all LMXBs are also made in GCs, but the only mechanism for the release of the field LMXBs is the tidal disruption of GCs. In this model, the predicted distribution of field LMXBs is more strongly peaked than that observed due to the high rates of GC destruction at the center of the galaxy. Although better data are needed, the comparison of the models with the present data indicate that at least half of the field LMXBs were made in situ.

\section{Acknowledgements}

I am very grateful to Greg Sivakoff and Adrienne Juett for helpful comments. This work was supported by the National Aeronautics and Space Administration through Chandra Award Numbers GO3-4099X, AR4-5008X, GO4-5093X, GO5-6081X, and GO5-6086X, and Hubble Space Telescope Award Number HST-GO-10003.01-A.

\section{References}

Angelini, L., Loewenstein, M., \& Mushotzky, R. F. 2001, ApJ 557, L35

Grindlay, J. E. 1984, $A d S p R$ 3, 19

Harris, W. E. 1991, ARA $\& A$ 29, 543

Hertz, P. \& Grindlay, J. E. 1983, ApJ 275, 105

Irwin, J. A. 2005, ApJ 631, in press

Jordán, A., Côté, P., Ferrarese, L., Blakeslee, J. P., Mei, S., Merritt, D., Milosavljević, M., Peng,

E. W., Tonry, J. L., \& West, M. J. 2004, ApJ 613, 279

Juett, A. M. 2005, ApJ 621, L25

Kundu, A., Maccarone, T. J., \& Zepf, S. E. 2002, ApJ 574, L5

Maccarone, T. J., Kundu, A., \& Zepf, S. E. 2003, ApJ 586, 814

Sarazin, C. L. 2005, ApJ submitted

Sarazin, C. L., Irwin, J. A., \& Bregman, J. N. 2000, ApJ 544, L101

-. 2001, ApJ 556, 533

Sarazin, C. L., Kundu, A., Irwin, J. A., Sivakoff, G. R., Blanton, E. L., \& Randall, S. W. 2003, ApJ 595, 743

Sivakoff, G. R., et al. 2005, ApJ submitted

White, R. E., Sarazin, C. L., \& Kulkarni, S. R. 2002, ApJ 571, L23 


\section{Discussion}

GHosh: Do we always assume that each GC contains a single X-ray binary? In other words, is the total luminosity associated with an X-ray source coincident with a globular cluster always thought to be that of a single binary? In particular, at the high-L end $\left(\sim 10^{39} \mathrm{erg} \mathrm{s}^{-1}\right)$, beyond the famous break in the XLF, is it possible that a GC has multiple unresolved sources?

SARAZIN: In general, we assume that all X-ray sources, including those in GCs, are single. Based on the X-ray luminosity function and fraction of GCs containing LMXBs, it would be unlikely that a GC would contain 2 bright LMXBs. (However, note that one Galactic GC does contain 2 active LMXBs). So, the sources are probably single, unless some subset of GC has a particularly high probability of having LMXBs.

KIM: In the case of the GC disruption for the formation of field LMXBs, the test of the SN proportionality may not work, because SN does not include those GC's disrupted.

SARAZIN: Yes. A worry with that test is that one has to assume that the original number of GCs is proportional to the present number. If different fractions of GCs were destroyed in different galaxies, this might be wrong. This is an advantage of the spatial distribution test I described.

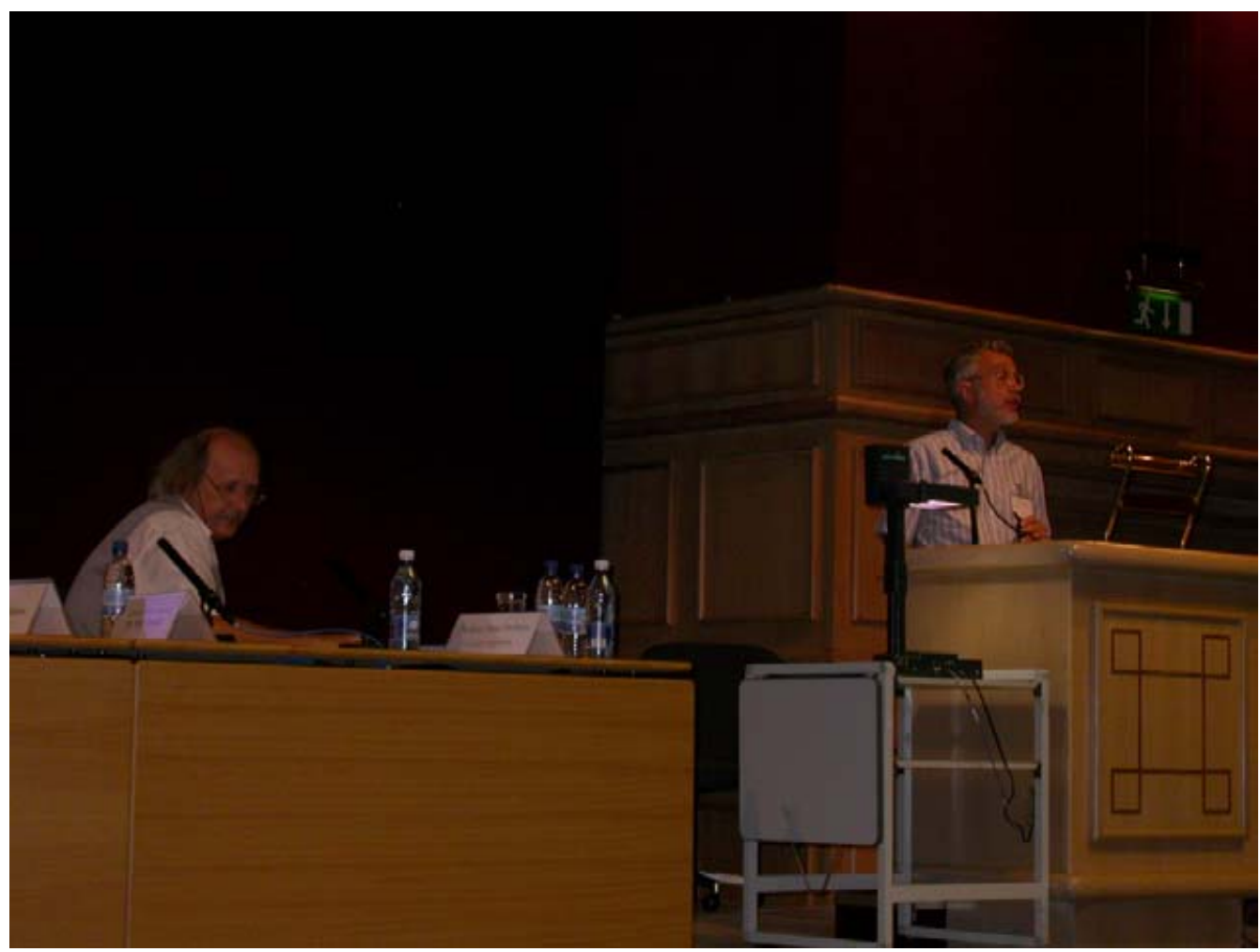

Chairman Vladimir Lipunov (left) following Craig Sarazin via a secret monitor. 